\title{
The application of nonlinear bistable detectors to DCT-domain watermarking schemes
}

\author{
Fabing Duan ${ }^{a}$ and Derek Abbott ${ }^{b}$ \\ ${ }^{a}$ Institute of Complexity Science, Qingdao University, Qingdao 266071, PR China; \\ ${ }^{b}$ Centre for Biomedical Engineering (CBME) and School of Electrical \& Electronic \\ Engineering, The University of Adelaide, Adelaide, SA 5005, Australia
}

\begin{abstract}
A DCT-domain watermarking scheme, based on nonlinear bistable detectors, is presented. A binary copyright character, i.e. watermark, is firstly reordered into a binary zig-zag sequence, and then mapped into pulse amplitude modulated waveforms. A certain desynchronization time delay can be arbitrarily placed into one code of the modulated signal, and is tolerated due to the superior robustness of nonlinear detectors over matched filters. The watermark signal is then embedded in a selected set of DCT coefficients of an image in a medium frequency range. The selected set of DCT coefficients is shuffled via the Arnold transform and looks more like background noise with respect to the watermark signal. The copyright character can be extracted by the nonlinear bistable detector without prior knowledge of the original image, i.e. blind watermark detection. Interestingly, a higher match between the original watermark character and the extracted one can be further achieved using a parallel array of bistable detectors via the mechanism of array stochastic resonance. Robustness of the proposed watermarking scheme is shown in the presence of noise, filtering, cropping and compression.
\end{abstract}

Keywords: Bistable detector, Watermark detection, Arnold transform, Array stochastic resonance

\section{INTRODUCTION}

Digital watermarking has recently received great attention as a tool for copyright protection. ${ }^{1-8,12,13}$ A digital watermark contains information about the copyright owner, the authorized consumer or other information need to be embedded. There are two main parts to building a strong watermark: the watermark structure and the insertion strategy. ${ }^{1}$ The strong watermark should be robust to various forms signal post processing, e.g. cropping and compression. The insertion strategy should elicit a convenient watermark detection procedure.

So far, there are two main proposed watermarking techniques, i.e. embedding the watermark in the spatial domain and the transformed domain of an original image. ${ }^{1-8,12,13}$ Now, the later method is preferable, e.g. embedding the watermark in DCT image domain. There are also two main watermark detection techniques according to the way the watermark is recovered from the possibly distorted version of the marked image, i.e. nonblind and blind watermark detection. ${ }^{1-3}$ The approach of extracting the embedded mark, without comparing the original and the marked images, will be referred to as the blind watermarking detection technique. Moreover, the original watermark is also not needed. ${ }^{7,8}$

In this paper, we propose a new blind DCT-domain watermarking scheme based on two important characteristics of nonlinear bistable detectors. Digital watermarking systems can be viewed as digital communication systems. ${ }^{6}$ Here, the watermark can be regarded as input information, while the DCT coefficients of an image can be thought of as background noise. In the course of the watermark detection, a nonlinear bistable system acts as a detector. ${ }^{9-11,14,15}$ The watermark structure and the insertion strategy are closely associated with characteristics of this nonlinear bistable detector.

This nonlinear bistable detector is a frequently used model for characterizing stochastic resonance phenomena in diverse scientific fields. ${ }^{9-11,14,15}$ Actually, many experimental physical devices demonstrating stochastic resonance effects possess a bistable characteristic. ${ }^{9,11}$ In the context of signal processing, this nonlinear phenomenon

Further author information: (Send correspondence to F. Duan)

F. Duan: E-mail: fabing1974@yahoo.com.cn;

D. Abbott: E-mail: dabbott@eleceng.adelaide.edu.au

Complex Systems II, edited by Derek Abbott, Tomaso Aste, Murray Batchelor, Robert Dewar,

Tiziana Di Matteo, Tony Guttmann, Proc. of SPIE Vol. 6802, 680215, (2008)

0277-786X/08/\$18 - doi: $10.1117 / 12.768057$

Proc. of SPIE Vol. $6802680215-1$ 
allows a weak signal to be enhanced by adding an optimal non-zero level of noise in some nonlinear systems. Naturally, this interesting observation triggered many significant studies in further analyzing the potential of such nonlinear systems for signal processing. ${ }^{9-11,14,15}$ Some promising results were also presented. ${ }^{10,11,14,15}$ Our recent studies of bistable systems indicate that (i) this system is robust in such degraded conditions as desynchronization ${ }^{10}$ or blind detection problems; ${ }^{11}$ (ii) a parallel array of bistable systems exhibits the array stochastic resonance phenomenon. ${ }^{14,15}$

Recent research suggests that bistable systems exhibit the phenomenon of stochastic resonance in the content of watermarking detection. ${ }^{12,13}$ In this letter, we also integrate this nonlinear bistable detector into the design of watermark scheme. The watermark consists of a binary copyright character. Next, this binary character is reordered into a zig-zag scan sequence. This sequence is then mapped into an one-dimensional pulse amplitude modulated (PAM) signal. Especially, this watermark signals is deliberately desynchronized by inserting a delay time at one arbitrary code. From the full-frame DCT domain of an original image, we select a set of DCT coefficients from the mid range of the frequency spectrum. These selected DCT coefficients undergo the Arnold transform for certain times, and behave more like background noise. Then, the watermark signal is superimposed on the disordered DCT coefficients, resulting in the marked DCT coefficients. In succession, the marked DCT coefficients return to their corresponding positions via the Arnold transform, and replace the selected set of DCT coefficients of the original image. After the inverse transform of the marked full-frame DCT coefficients, the marked image will be obtained. The proposed watermarking scheme is tested on some common forms of interference and distortion: additive Gaussian noise, histogram equalization, JPEG compression, low pass and median filtering, geometric cropping, salt \& pepper noise and multiplicative speckle noise. Given various corrupted marked images, the watermark can be recovered by reversing the watermark embedding procedure. Then, it is applied to an isolated bistable detector or a parallel array of nonlinear bistable detectors for extracting the watermark, i.e. the copyright character. Finally, this watermark scheme is evaluated by measuring the similarity between the extracted character and the original one. Due to the special watermark structure, this method makes the nonlinear bistable detector especially suited to the problem compared to a matched filter. Interestingly, we find that array stochastic resonance, by a parallel array of nonlinear bistable detectors, can further improve the similarity measure to a higher level than an isolated bistable detector does. It is noted that the internal array noise plays a constructive role in the watermark detection and acts as an operational tool for improving the detectability of detectors. This application of bistable systems to DCT domain watermarking detection indicates that nonlinear systems are potential signal processors, and stochastic resonance is a useful mechanism in signal processing field.

\section{WATERMARK EMBEDDING}

\subsection{Structure of watermark}

In this paper, a black-and-white copyright character $S$ of size $29 \times 29$, as shown in Fig. 1 (a), is employed as a binary watermark. Since 0 s and 1 s correspond to black and white pixels, this copyright character $S$ contains $L=841$ bits. This two-dimensional binary image $S$ is firstly reordered into an one-dimensional zig-zag sequence, such as in the JPEG compression algorithm. ${ }^{2}$ Next, we modulate this binary zig-zag sequence onto a PAM signal with amplitude $\pm A$ and bit interval $T_{b}$. Ten examples of the PAM waveforms are shown in Fig. 1 (b). Each waveform lasts for a bit interval $T_{b}$, but an arbitrary waveform is deliberately delayed by $0.5 T_{b}$, as shown in Fig. 1 (b). This kind of desynchronized PAM signal is specially designed for the watermarking detection scheme. In succession, the PAM waveform is sampled with a sampling time $0.01 T_{b}$. Thus, each pulse contains 100 sample values and a sample signal vector $X=\left\{x_{1}, x_{2}, \cdots, x_{84100}\right\}$ is obtained. Note that the last PAM waveform is a half bit interval having 50 sampled points. In this way, a copyright character $S$ will be transformed into a watermark vector $X$ to be embedded into the DCT domain of an image.

\subsection{Watermark casting}

In this step, the $512 \times 512$ DCT coefficients for a $512 \times 512$ gray-scale image $I$, as shown in Fig. 2 (a), are computed. The DCT coefficients are also reordered into a zig-zag scan, and the first DCT coefficient should be the DC term. In line with some important casting methods, ${ }^{1,2,12,13}$ the coefficients from the 80001 th to the 
(a)

\section{DA}

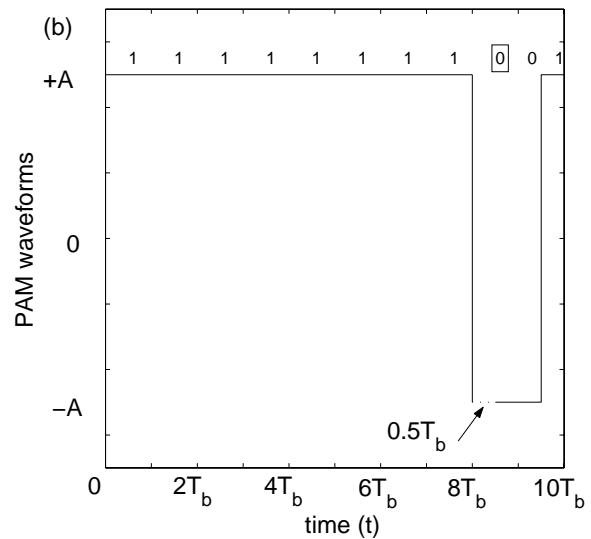

Figure 1. (a) Binary copyright character $S$ with size of $29 \times 29$. (b) Examples of PAM waveform encoded by the zig-zag version of the copyright character. Here, the pulse amplitude is $\pm A$ and the bit interval is $T_{b}$. One code of bit 0 is deliberately desynchronized by a delay time of $0.5 T_{b}$, as the arrow indicated.

(a)

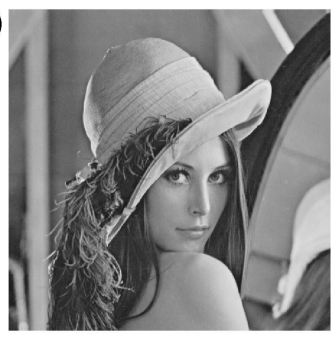

(b)

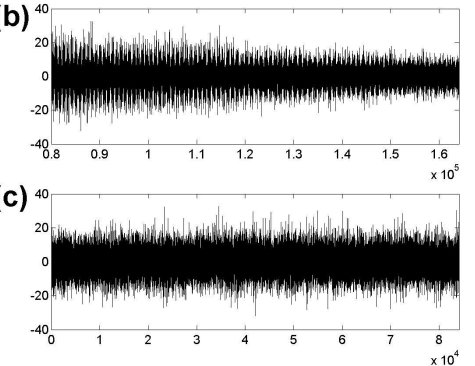

(d)

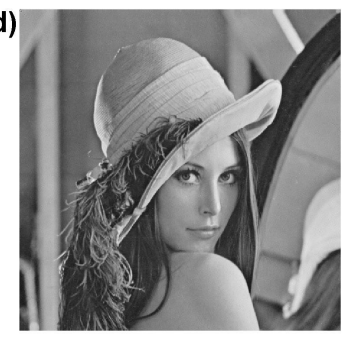

Figure 2. (a) Original $512 \times 512$ image 'Lena'. (b) Selected set of zig-zag scan of DCT coefficients. (c) After Arnold transform, the shuffled DCT coefficients sequence of $T$. (d) Marked image 'Lena'. Here, $A=4$ with the unit of the DCT coefficient.

164100th positions are taken out according to the above zig-zag sequence of the DCT spectrum, as shown in Fig. 2 (b). There are 84100 DCT coefficients in total to be modified.

In this paper, when considering stochastic resonance, we take the binary information as the input signal, and view the selected DCT coefficients as noise. However, as shown in Fig. 2 (b), the selected selected DCT coefficients are not as random as background noise. For example, the variance of coefficients reduces. Here, we reshape the selected selected DCT coefficients into a $290 \times 290$ matrix. Then, this reordered DCT coefficient matrix is shuffled by the Arnold transform ${ }^{8}$

$$
\left[\begin{array}{l}
x^{\prime} \\
y^{\prime}
\end{array}\right]=\left[\begin{array}{ll}
1 & 1 \\
1 & 2
\end{array}\right]\left[\begin{array}{l}
x \\
y
\end{array}\right](\bmod 290),
$$

where $x, y \in[1,2, \cdots, 290]$ are the pixel coordinates of the reshaped DCT coefficients, and $x^{\prime}, y^{\prime} \in[1,2, \cdots, 290]$ correspond to the shuffled DCT coefficients. Here, the Arnold transform iteration is $k=110$. If the shuffled DCT coefficients continue to undergo $m=100$ iterations of Arnold transform, the DCT coefficients will be optimally reshaped. This indicates $p=k+m$ Arnold transforms for a $290 \times 290$ DCT coefficients matrix is optimal. ${ }^{8}$

Next, the shuffled $290 \times 290 \mathrm{DCT}$ coefficients are reordered into a zig-zag sequence $T=\left\{t_{1}, t_{2}, \cdots, t_{84100}\right\}$, as plotted in Fig. 2 (c). $T$ is more like a random noise sample than the original zig-zag scan of Fig. 2 (b). Then, the input signal vector $X$ is added to the zig-zag sequence of $T$. The marked DCT coefficient sequence $T^{\prime}$ is given as

$$
T^{\prime}=T+X .
$$


Finally, the modified sequence $T^{\prime}=\left\{t_{1}^{\prime}, t_{2}^{\prime}, \cdots, t_{84100}^{\prime}\right\}$ is reinserted in the inverse zig-zag scan. Then, the $290 \times 290$ DCT coefficients are embedded, and undertake the residual $m=100$ Arnold transform iterations. We replace the $290 \times 290$ embedded DCT coefficients with the original ones in the full-frame DCT domain, and perform a $512 \times 512$ inverse DCT transform. The wartermarked image $I^{\prime}$ is then obtained, as shown in Fig. 2 (d). The peak signal-to-noise (PSNR) is calculated as $40.75 \mathrm{~dB}$.

\section{WATERMARK DETECTION AND SIMILARITY}

The marked image $I^{\prime}$ is possibly corrupted by some signal processing algorithms, e.g. compression, and becomes a corrupted image $I^{*}$. The $512 \times 512 \mathrm{DCT}$ transform is applied to the watermarked image $I^{*}$. Then, the $512 \times 512$ DCT coefficients embedded with watermarks can be obtained by reversing the steps of watermark casting in Sec. 2. It is reordered into a zig-zag sequence and coefficients from 80001th to the 164100th positions are selected. These 84100 coefficients are reshaped into a $290 \times 290$ matrix via the inverse of the zig-zag procedure, and the formed matrix undergoes 110 iterations of the Arnold transform. Then, this shuffled matrix is reordered into the zig-zag sequence $T^{*}=\left\{t_{1}^{*}, t_{2}^{*}, \cdots, t_{84100}^{*}\right\}$ to be detected for revealing the watermark. In order to extract the recovered binary watermarked image $S^{*}$, we utilize two alternative detection strategies as follows.

\subsection{An isolated nonlinear bistable detector}

In this detection task, we apply $T^{*}$ to a nonlinear bistable detectors given as ${ }^{10,11,15}$

$$
\tau_{a} \frac{d x(t)}{d t}=x(t)-\frac{x^{3}(t)}{X_{b}^{2}}+T^{*}(t)
$$

with detector parameters $\tau_{a}>0$ and $X_{b}>0$. Here, the differential equation of Eq. (3) is written as continuous form, and the vector $T^{*}$ is represented as a continuous input signal $T^{*}(t)$. In this letter, we numerically integrate Eq. (3) using Euler-Maruyama discretization with a sampling time step $\Delta t \ll T_{b} .{ }^{10,11,14,15}$ Throughout the paper, we adopt $\Delta t=0.01 T_{b}$. The output $x(t)$ is sampled for recovery of the watermark.

\subsection{A parallel array of nonlinear bistable detectors}

In our previous work in the area of array stochastic resonance, ${ }^{15}$ we have reported that signal-to-noise ratio gain exceeding unity is achievable when a parallel array of bistable dynamical subsystems is subjected to a noisy subthreshold or suprathreshold input. Here, an uncoupled parallel array of bistable detectors is also considered for obtaining an improved binary mark image. Zero-mean Gaussian white noise $\eta_{i}(t)$, together with and independent of $T^{*}(t)$, is applied to each detector of the parallel array of size $N$. The $N$ array noise terms $\eta_{i}(t)$ are mutually independent and have autocorrelation $\left\langle\eta_{i}(t) \eta_{i}(0)\right\rangle=D_{\eta} \delta(t)$ with a same noise intensity $D_{\eta}$. The internal state $x_{i}(t)$ of each bistable detector is governed by

$$
\tau_{a} \frac{d x_{i}(t)}{d t}=x_{i}(t)-\frac{x_{i}^{3}(t)}{X_{b}^{2}}+T^{*}(t)+\eta_{i}(t)
$$

for $i=1,2, \ldots, N$. Their outputs are averaged and the response of the array is given as

$$
y(t)=\frac{1}{N} \sum_{i=1}^{N} x_{i}(t) .
$$

Then, the response $y(t)$ of Eq. (5) is subsequently sampled in order to recover the watermark.

\subsection{Recovering procedure and the similarity measure}

We introduce the detection scheme as follows: the output $x(t)$ of Eq. (3) or the average output $y(t)$ of Eq. (5) is sampled at $j T_{b}$ for $j=1,2, \ldots$, resulting in a successive sampled values of $Y_{j} .{ }^{10,11,14,15}$ Note that the sample points take on the corresponding numerical values at $j T_{b}$, regardless of the desynchronization time. This sampling method has a weak effect on the bistable detector, but a deleterious effect on a matched filter. ${ }^{10,11}$ Thus, the bistable detector is utilized as a novel robust detector in this watermark scheme. Next, $Y_{j}$ are compared with 

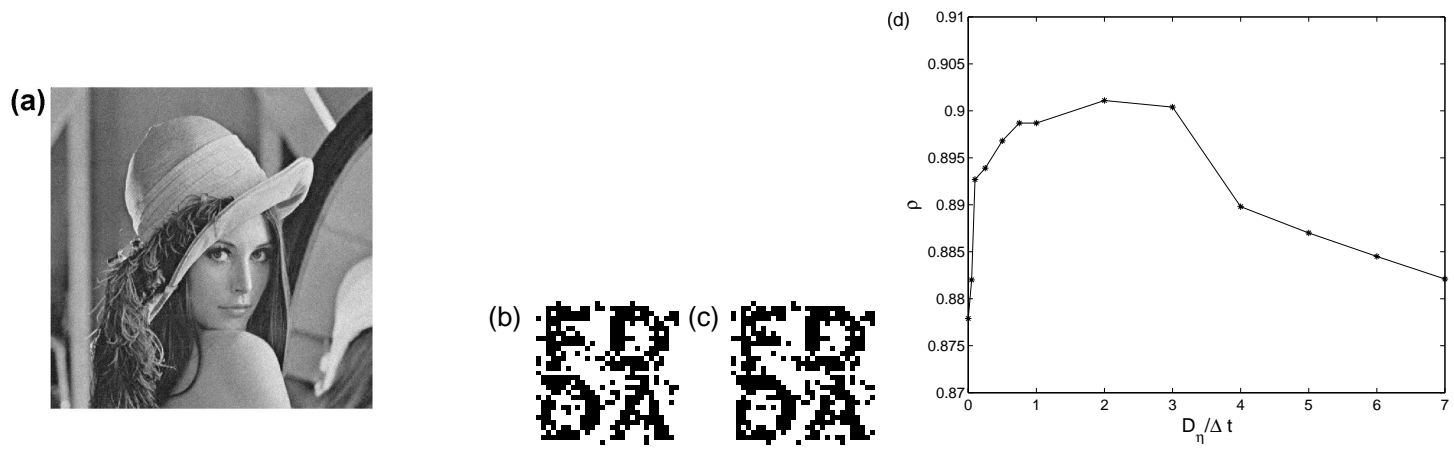

Figure 3. (a) Watermarked image 'Lena' corrupted by Gaussian noise with variance $\sigma^{2}=100$. (b) The recovered binary image detected by the isolated bistable detector, and (c) recovered image detected by the array of bistable detectors with optimal noise intensity $D_{\eta} / \Delta t=200$. (d) The resonance-like curve of $\rho$ versus $D_{\eta}$ for the array of bistable detector. Here, $\tau_{a}=T_{b} / 150, X_{b}=3.75 A$ and the array size $N=60$.

the decision threshold $\ell$ for selecting binary values 0 or 1 . If $Y_{j}>\ell$, the recovered mark is read as a digital 1 , otherwise a digital 0. Here, we utilize the hard thresholding method of $\ell=0 .{ }^{11}$ Now, this system of Eq. (3) or Eq. (4) with input binary signal and output reading binary bits, can be viewed as an information channel transmitting binary data.

Following the above detection scheme, we will obtain a recovered binary sequence. This binary sequence is then reshaped by the inverse zig-zag scan, and the recovered binary image of copyright character $S^{*}$ can be formed. The similarity $\rho$ between the original character $S$ and the recovered one $S^{*}$ is measured by

$$
\rho=\frac{S \cdot S^{*}}{S^{*} \cdot S^{*}}=\frac{\sum \sum s s^{*}}{\sum \sum s^{* 2}}
$$

where $s$ and $s^{*}$ are the pixel values of $S$ and $S^{*}$, respectively. We compare $\rho$ with a threshold to decide how similar the recovered mark $S^{*}$ is to the original $S$. The threshold has a range from zero to unity, and the exact value of the threshold depends on the requirements. The larger $\rho$ is, the more similar $S^{*}$ is to $S$.

\section{EXPERIMENTAL RESULTS}

In order to evaluate the proposed watermarking scheme, the $29 \times 29$ copyright character is embedded into the grey-scale standard image 'Lena'. Several common signal processing techniques and geometric distortions are applied to these marked images to evaluate if the nonlinear detector of Eq. (3) or Eq. (4) can reveal the embedded mark well or not. The similarity measure will enable the robustness of this watermarking scheme to be evaluated.

In this paper, main experimental descriptions of the standard image 'Lena' are presented. The bistable detector has tunable parameters $\tau_{a}$ and $X_{b}$. The $\tau_{a}$ and $X_{b}$ parameter tuning method is described by Duan et al. ${ }^{11}$ The noise intensity $D_{\eta}$ is also a tunable parameter for the array of bistable detectors. ${ }^{15}$

\subsection{Adding Gaussian noise}

Zero-mean Gaussian noise with variance $\sigma^{2}$ is introduced to corrupt the marked image $I^{\prime}$. As the variance $\sigma^{2}$ increases, the image degradation is severer. An example corrupted image is illustrated in Fig. 3 (a) for Gaussian noise variance $\sigma^{2}=100$. The PSNR of the corrupted image $I^{*}$ is $27.86 \mathrm{~dB}$. The isolated bistable detector detects the binary mark image with similarity $\rho=0.8770$. While the array of nonlinear detectors of Eq. (5) gives $\rho=0.9011$ at an optimal noise intensity $D_{\eta} / \Delta t=200$. Both recovered binary watermark images $S^{*}$ are shown in Fig. 3 (b) and (c), respectively. Note that $D_{\eta}$ is an independent tunable parameter and determined in the following way: Upon increasing $D_{\eta}$ from zero, as shown in Fig. 3 (d), $\rho$ shows a resonance-like curve; We select the highest value of similarity $\rho=0.9011$, and then obtain the corresponding optimal value of $D_{\eta} / \Delta t=200$.

As the marked image $I^{\prime}$ is corrupted at a different Gaussian noise variance $\sigma^{2}$, we follow the above way of tuning noise intensity $D_{\eta}$. The corresponding optimal similarity $\rho$ is plotted in Fig. 4 for the array of bistable 


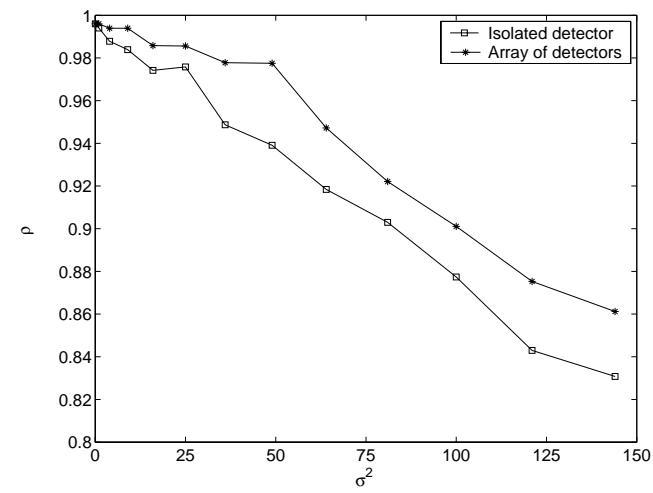

Figure 4. Similarity $\rho$ as a function of Gaussian noise variance $\sigma^{2}$. Each point of $\rho$ of the array of bistable detectors is obtained by optimizing the noise intensity $D_{\eta}$ in the way of the example of Fig. 3 .
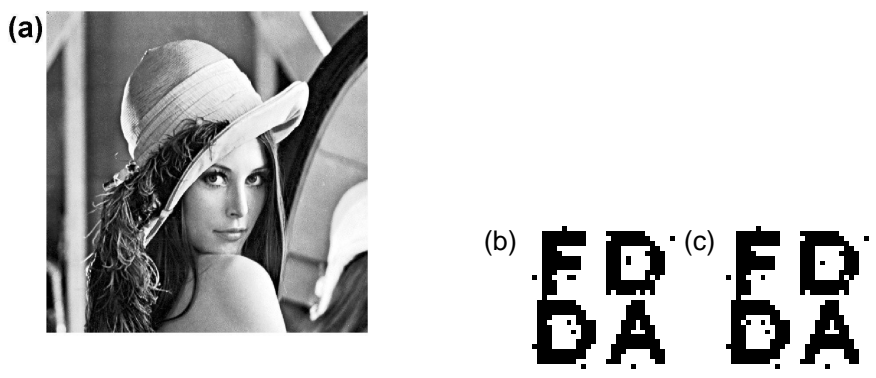

Figure 5. (a) Watermarked image 'Lena' after histogram equalization. The recovered binary image via (b) the isolated bistable detector, and (c) the array of bistable detectors with optimal noise intensity $D_{\eta} / \Delta t=50$. Here, $\tau_{a}=T_{b} / 150$, $X_{b}=3.75 A$ and the array size $N=60$.

detectors (stars). For comparison, the isolated bistable detector (squares) is also drawn in Fig. 4 . It is seen that the array noise $\eta_{i}(t)$ improves the similarity $\rho$ via the mechanism of array stochastic resonance. ${ }^{15}$

\subsection{Histogram equalization}

As shown in Fig. 5 (a), the marked image $I^{\prime}$ is operated by histogram equalization into $I^{*}$ with the PSNR being $19.04 \mathrm{~dB}$. The isolated bistable detector presents the mark $S^{*}$, as shown in Fig. 5 (b), with the similarity $\rho=0.9836$. Next, we adopt an array of nonlinear detectors of Eq. (5) for the watermark detection. The array size is $N=60$. The largest $\rho$ is 0.9857 , and the corresponding optimal noise intensity $D_{\eta} / \Delta t=50$. The recovered watermark $S^{*}$ is plotted in Fig. 5 (c).

\subsection{JPEG compression}

The JPEG compression algorithm is one of the most severe distortion that the watermark meets. We applied JPEG coding with decreasing quality and $0 \%$ smoothing to the marked image $I^{\prime}$. Obviously, the measure of $\rho$ is plotted in Fig. 6 as the monotonic increasing function of JPEG compressing quality. However, the array of bistable detectors presents a much better result than an isolated bistable detector does.

An example compressing version of image with $75 \%$ quality and $0 \%$ smoothing is shown in Fig. 7 (a) (The PSNR is $36.83 \mathrm{~dB}$ ), and the corresponding recovered binary characters $S^{*}$ are plotted in Fig. 7 (b) and (c), respectively. Here, Fig. 7 (b) is similar to the original binary character with $\rho=0.8200$, whereas Fig. 7 (c) is with $\rho=0.8620$. The detected mark of the array of the bistable detectors looks more better. 


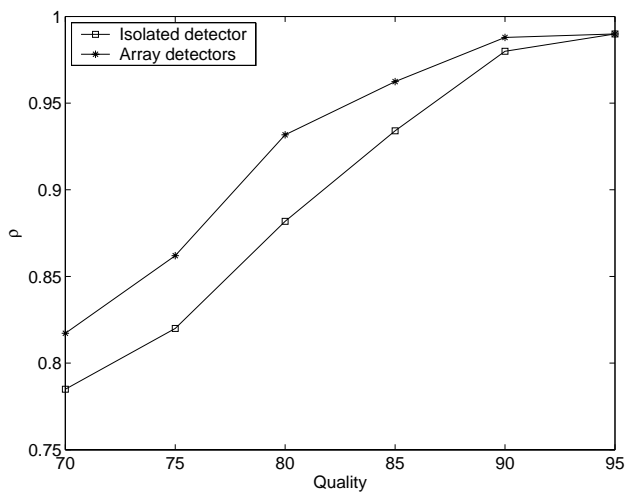

Figure 6. Similarity $\rho$ as a function of JPEG compression quality. Here, the compression quality means 100 minus the compression ratio.

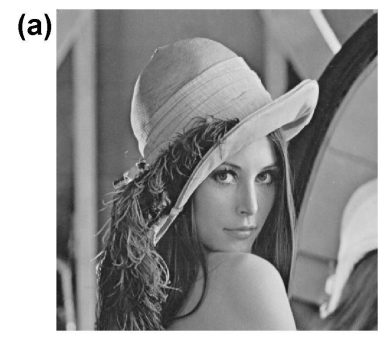

(b)

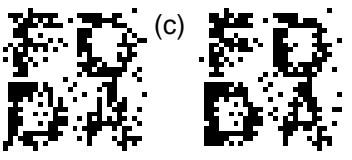

Figure 7. (a) JPEG compressed version of the marked image 'Lena' with $75 \%$ quality and $0 \%$ smoothing. The recovered binary image via (b) the isolated bistable detector, and (c) the array of bistable detectors with optimal noise intensity $D_{\eta} / \Delta t=100$. Here, $\tau_{a}=T_{b} / 150, X_{b}=3.75 A$ and the array size $N=60$.

\subsection{Low pass filtering and high pass filtering}

The watermarked image $I^{\prime}$ is filtered by a low pass filter with window size $2 \times 2$, as shown in Fig. 8 (a). The PSNR is $29.72 \mathrm{~dB}$. The corresponding recovered binary characters $S^{*}$ are also illustrated in Fig. 8 (b) and (c), with similarity $\rho=0.8862$ and $\rho=0.8917$, respectively.

The watermarked image $I^{\prime}$ is also filtered by a high pass filter with window size $4 \times 4$, as shown in Fig. 9 (a). The PSNR is $30.51 \mathrm{~dB}$. The corresponding recovered binary characters $S^{*}$ are also illustrated in Fig. 9 (b) and (c), with similarity $\rho=0.9816$ and $\rho=0.9827$, respectively. Note that this watermarking scheme failed

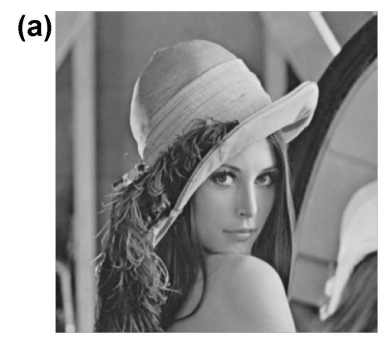

(b)

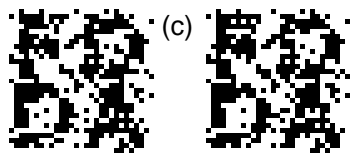

Figure 8. (a) The marked image 'Lena' filtered by a low pass filter with window size $2 \times 2$. The recovered binary image $S^{*}$ via (b) the isolated bistable detector, and (c) the array of bistable detectors with optimal noise intensity $D_{\eta} / \Delta t=5$. Here, $\tau_{a}=T_{b} / 50, X_{b}=0.75 A$ and the array size $N=60$. 

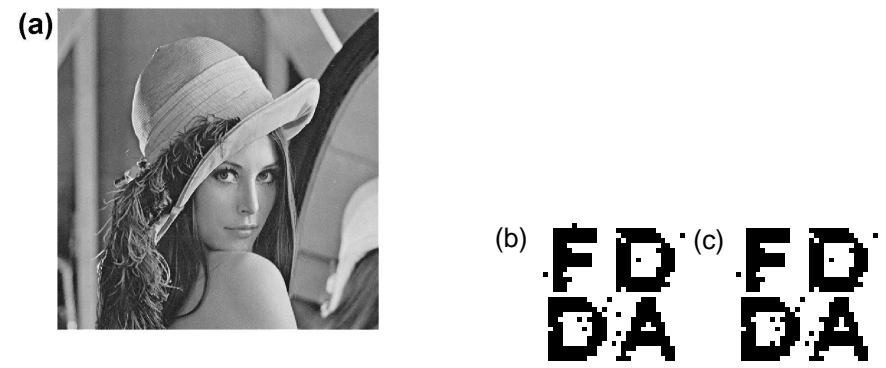

Figure 9. (a) The marked image 'Lena' filtered by a high pass filter with window size $4 \times 4$. The recovered binary image $S^{*}$ via (b) the isolated bistable detector, and (c) the array of bistable detectors with optimal noise intensity $D_{\eta} / \Delta t=100$. Here, $\tau_{a}=T_{b} / 50, X_{b}=1.25 A$ and the array size $N=60$.

(a)

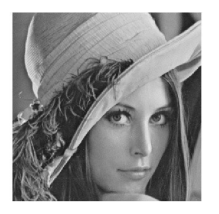

(b)

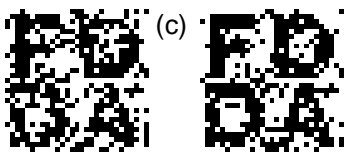

Figure 10. (a) The marked image 'Lena' after cropping as $300 \times 300$ subpart. The recovered binary image $S^{*}$ via (b) the isolated bistable detector, and (c) the array of bistable detectors with optimal noise intensity $D_{\eta} / \Delta t=200$. Here, $\tau_{a}=T_{b} / 150, X_{b}=3.75 A$ and the array size $N=60$.

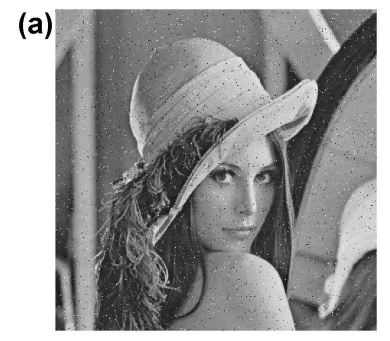

(b)

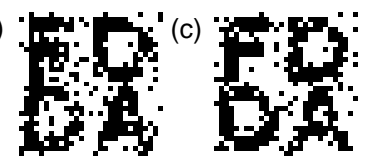

Figure 11. (a) The marked image 'Lena' added by salt \& pepper noise with noise density 0.02 . The recovered binary image $S^{*}$ via (b) the isolated bistable detector, and (c) the array of bistable detectors with optimal noise intensity $D_{\eta} / \Delta t=50$. Here, $\tau_{a}=T_{b} / 30, X_{b}=3.75 A$ and the array size $N=60$. 


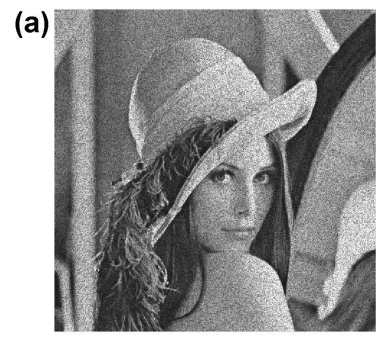

(b)

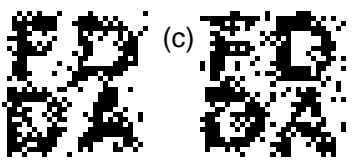

Figure 12. (a) The marked image corrupted by multiplicative speckle noise with noise variance 0.05 . The recovered binary image by (b) the isolated bistable detector and (c) the array of bistable detectors with optimal noise intensity $D_{\eta} / \Delta t=1000$. Here, $\tau_{a}=T_{b} / 10, X_{b}=3.75 A$ and the array size $N=60$.

under the median filter. The main reason is the mark $S$ is embedded in median frequency DCT domain.

\subsection{Geometric cropping}

We cropped the watermarked image $I^{\prime}$ as $300 \times 300$ subpart $I^{*}$, as shown in Fig. 10 (a). The PSNR is 7.21 dB. The corresponding recovered binary characters $S^{*}$ are illustrated in Fig. 10 (b) and (c), with similarity $\rho=0.8502$ and $\rho=0.9008$, respectively. It is observed that the addition of array noise is obviously helpful to the detectability of nonlinear detectors.

\subsection{Salt \& pepper noise}

We corrupted the watermarked image with salt \& pepper noise with noise density 0.02, as shown in Fig. 11 (a). The PSNR is $22.34 \mathrm{~dB}$. The corresponding recovered binary characters are also illustrated in Fig. 11 (b) and (c), with similarity $\rho=0.8985$ and $\rho=0.9191$, respectively.

\subsection{Multiplicative speckle noise}

We corrupted the watermarked image with multiplicative speckle noise with noise variance 0.05 , as shown in Fig. 12 (a). The PSNR is $18.82 \mathrm{~dB}$. The corresponding recovered binary characters are also illustrated in Fig. 12 (b) and (c), with similarity $\rho=0.8766$ and $\rho=0.8802$, respectively.

\section{CONCLUSION}

In this paper, we propose a new blind watermarking scheme for digital gray scale images operating in the DCT domain: a binary character is embedded in a selected set of DCT coefficients and a nonlinear detector is employed for watermark detection. The character mark is reordered in a zig-zag sequence, and this sequence is then modulated as a PAM signal. This watermark signal is delibertely desynchronized by a delay time at one arbitrary code to test robustness. The full-frame DCT coefficients are also arranged in a zig-zag scan, and extracted in the median frequency domain. The extracted set of DCT coefficients is further shuffled by the Arnold transform, and acts as the background noise for the watermark signal. The watermark signal is then added to the shuffled DCT coefficients. Watermark detection employs a bistable detector and a parallel array of bistable detectors, since the nonlinear bistable detector is robust to the delay time in the PAM watermark signal. Experimental results demonstrate that this watermark scheme is robust to several signal processing techniques, such as additive Gaussian noise, histogram equalization, JPEG compression, low pass and high pass filtering, image cropping, salt \& pepper noise and multiplicative speckle noise. Interestingly, the array of bistable detectors has a tunable parameter of array noise for improving the similarity of the recovered character to the original one. This mechanism is referred to array stochastic resonance. Future research of this scheme deserves deeper investigation in embedding the binary information in DWT coefficients. 


\section{ACKNOWLEDGMENTS}

This work is sponsored by NSFC (No. 60602040), Taishan Scholar CPSP, the SRF for ROCS, SEM and PhD PFME of China (No. 20051065002). Funding from the Australian Research Council (ARC) is gratefully acknowledged.

\section{REFERENCES}

1. I.J. Cox, J. Kiliant, T. Leighton and T. Shamoon, "Secure spread spectrum watermarking for multimedia," IEEE Trans. on Image Processing 6, pp. 1673-1687, 1997.

2. M. Barni, F. Bartolini, V. Cappellini and A. Piva, "A DCT-domain system for robust image watermarking," Signal Processing 66, pp. 357-372, 1998.

3. M. Barni, F. Bartolini, A.D. Rosa and A. Piva, "Capacity of full frame DCT image watermarks," IEEE Trans. on Image Processing 9, pp. 1450-1455, 2000.

4. E.Y. Lam and J.W. Goodman, "A mathematical analysis of the DCT coefficient distributuions for images," IEEE Trans. on Image Processing 9, pp. 1661-1666, 2000.

5. S. Stanković, I. Djurović, R. Herpers and L. Stanković, An approach to optimal watermark detection, Int. J. Electron. Commun., 57, pp. 355-357 (2003).

6. S. baudry, J.F. Delaigle, B. Sankur, B. Macq and H. Maître, "Analyses of error correction strategies for typical communication channels in watermarking," Signal Processing 81, pp. 1239-1250, 2001.

7. I. Katzenbeisser, S. Petitcolas, A.P. Fabien, Information Hiding Techniques for Steganography and Digital Watermarking, Artech House, Inc, 2000.

8. M. Barni, F. Bartolini, Watermarking Systems Engineering, Marcel Dekker, Inc, 2004.

9. L. Gammaitoni, P. Hänggi, P. Jung, and F. Marchesoni, "Stochastic resonance," Rev. Mod. Phys. 70, pp. 233-287, 1998.

10. D Rousseau, J.R. Varela, F. Duan, F. Chapeau-Blondeau, "Evaluation of a nonlinear bistable filter for binary signal detection," Int. J. Bifurcation and Chaos 15, pp. 667-679, 2005.

11. F. Duan, D. Abbott, and Q. Gao, "Evaluation of bistable systems versus matched filters in detecting bipolar pulse signals," Fluctuation and Noise Lett. 5, pp. L127-L142, 2005.

12. S. Sun and P. Qiu, "Algorithm of digit watermarking based on parameter-induced stochastic resonance," J. Communications 26, pp. 48-55, 2005.

13. G. Wu and Z. Qiu, "A novel watermarking scheme based on stochastic resonance," IEEE the 8th International Conference on Signal Processing 2, pp. 16-20, 2006.

14. F. Chapeau-Blondeau, and D. Rousseau, "Enhancement by noise in parallel arrays of sensors with power-law characteristics," Phys. Rev. E 70, 060101(R), 2004.

15. F. Duan, F. Chapeau-Blondeau, and D. Abbott, "Noise improvement of SNR gain in parallel array of bistable dynamic systems by array stochastic resonance," Electronics Lett. 42, pp. 1008-1009, 2006. 